\title{
ORIGINAL ARTICLE \\ Type, intensity and duration of daily physical activities performed by adults with spinal cord injury
}

\author{
M-J Perrier, MJ Stork, KA Martin Ginis and The SHAPE-SCI Research Group
}

\begin{abstract}
Study design: Secondary analysis of cross-sectional data.
Objectives: To describe and compare (1) self-reported intensities and durations of specific types of daily physical activities and (2) minutes per day spent on daily physical activities across key demographic groups.

Setting: Community (Ontario, Canada).

Methods: Participants were 695 adults with spinal cord injury $\left(\mathrm{SCl} ; 76 \%\right.$ male, $M_{\text {age }}=46.81 \pm 13.41$ years, $M_{\text {years post injury }}=15.19$ \pm 11.10 years). Daily activities were assessed over the telephone using the Physical Activity Recall Assessment for People with SCl. Multivariate analyses of variance (MANOVA) were computed to test for differences in intensities and durations of different daily activities (objective 1) and between-group differences in minutes per day of daily activities (objective 2).

Results: Overall, participants reported $127.92 \pm 142.79$ min per day of daily physical activities with significantly more time spent in mild intensity (78.93 $\pm 104.62 \mathrm{~min}$ per day) than moderate- $(40.23 \pm 68.71$ min per day) or heavy-intensity activities $(8.75 \pm 24.53$ min per day). Four patterns emerged with respect to type, duration and intensity, with some activities being typically performed at lighter or heavier intensities than others. There were significant differences in minutes per day of activity intensity and duration between groups based on education, injury severity and mode of mobility $(P<0.05)$.

Conclusion: Given that some groups were more likely to engage in moderate-heavy-intensity activities, and some activities were more likely to be performed at moderate-heavy intensities, interventions that target key groups to increase certain daily activities may be one strategy to enhance overall physical activity participation among people with $\mathrm{SCl}$.
\end{abstract}

Spinal Cord (2017) 55, 64-70; doi:10.1038/sc.2016.86; published online 7 June 2016

\section{INTRODUCTION}

People with spinal cord injury (SCI) have an increased risk of chronic disease when compared with the general population. For example, people with SCI have 2.5 greater odds of developing type II diabetes ${ }^{1}$ and other cardiovascular disease risk factors when compared with the general population. ${ }^{2}$ This risk can be mitigated by engaging in leisuretime physical activity (LTPA), physical activity that individuals choose to do in their spare time, such as playing a sport, playing with one's children, going out for a wheel or lifting weights at the gym. ${ }^{3-5}$ For example, engaging in at least 150 min per week of LTPA for 3 months was associated with a lower fat mass, lower $c$-reactive protein, and serum insulin and leptin levels among men with cervical SCI. ${ }^{4}$ Despite these benefits, an estimated $50 \%$ of people with SCI accrue absolutely no LTPA. $^{6}$ Similar estimates of inactivity have been reported in the United Kingdom and the United States $(53 \%$ and $47 \%$, respectively). ${ }^{7,8}$ Low levels of LTPA can be attributed, at least in part, to barriers such as limited accessibility to recreational facilities, fewer opportunities to participate in sport and exercise, and lack of knowledge regarding how or where to participate. ${ }^{2,9,10}$ As such, there may be merit to encouraging participation in alternative forms of physical activity, such as daily activities that require energy expenditure (for example, cleaning and mobility), to increase the amount of overall physical activity that people with SCI accrue.
Preliminary research has shown an association between certain daily activities (that is, activities other than those categorised as LTPA), and quality of life and fitness. Hetz et al. ${ }^{11,12}$ found positive correlations between minutes per day spent on certain daily activities (for example, cleaning) and aerobic fitness, as well as negative associations between increased daily activity and chronic disk risk indicators (for example, total cholesterol), even when controlling for time spent on LTPA. Furthermore, Collins et al. ${ }^{13}$ found that some daily activities such as wheeling for transportation on uneven terrain require several metabolic equivalent units - even more than some forms of LTPA. Thus, some physical activities other than LTPA may confer fitness and quality of life benefits to people with SCI.

However, these previous studies have not considered a comprehensive list of daily activities, nor were the intensity of the activities taken into consideration. SCI research indicates that to confer fitness benefits LTPA must be performed for at least 20 min, twice per week, at a moderate-to-heavy intensity. ${ }^{14}$ Presumably, daily activities would also need to be performed at a minimum intensity and duration in order to confer health benefits; however, a minimum duration for daily activities has yet to be determined. ${ }^{15}$ Moreover, LTPA participation differs as a function of demographic and injury characteristics, such as gender and mode of mobility, suggesting a need for tailored interventions to target those who are at greater risk of being insufficiently active. ${ }^{6}$ When considering how to encourage people

Department of Kinesiology, McMaster University, Hamilton, Ontario, Canada

Correspondence: Dr M-J Perrier, The SHAPE-SCI Research Group, Department of Kinesiology, McMaster University, 1280 Main Street West, Hamilton, Ontario L8S 4K1, Canada. E-mail: perrier@mcmaster.ca

Received 18 February 2016; revised 6 April 2016; accepted 10 April 2016; published online 7 June 2016 
with SCI to be more physically active, it would be valuable to know which daily activities are typically performed at the greatest intensities and durations, and by whom, as these activities may be ideal to promote as strategies to enhance one's overall level of physical activity.

Given these considerations, the first objective of this study was to describe and compare the intensities and durations of daily activities that are typically reported by people with SCI. As this objective was descriptive in nature, no specific hypotheses were made. Our secondary objective was to describe and compare the daily duration and intensity of daily activities across key demographic groups. On the basis of previous research on LTPA, ${ }^{6}$ we hypothesised that (1) there would be no group differences as a function of education, ethnicity or marital status, but (2) on the basis of the finding that women accrued more minutes of certain daily activities than men (for example, cleaning), women would accrue more minutes per day of daily activities than men. ${ }^{12}$ On the basis of previous research on LTPA, ${ }^{6}$ we hypothesised that there would be between-group differences for injury severity and mode of mobility groups, such that people with less severe paraplegia (for example, American Spinal Injury Association Impairment Scale (AIS) D) would accrue more minutes of activity at greater intensities than those with more severe injuries (for example, AIS A-C, any tetraplegia) and those using gait aids would accrue more minutes of daily activities at greater intensities than those using a manual or power chair.

\section{METHODS}

This study is a secondary analysis of the Study of Health and Activity in People with Spinal Cord Injury (SHAPE-SCI) data, ${ }^{16}$ specifically focusing on daily activities data. Although detailed information about study methods has been published, we briefly summarise below the methods used in SHAPE-SCI.

\section{Participants and data collection}

Participants were 695 people with a traumatic SCI, at least 1 year post injury and living in the community $\left(76 \%\right.$ men, $M_{\text {age }}=46.81 \pm 13.41, M_{\text {years post }}$ injury $=15.19 \pm 11.10$ ). Participants were required to speak English and be able to provide informed, voluntary consent (that is, age $\geqslant 18$ years, no cognitive impairment). Information about their demographics, injuries and daily physical activities, including both LTPA and other daily activities, was collected in structured telephone interviews. ${ }^{16}$ All methods were approved by the Research Using Ethics Board at the participating institutions. The measures included in the present analysis are described below.

\section{Daily activities}

Detailed daily activities data including type, intensity and duration were collected using the Physical Activity Recall Assessment for People with SCI (PARA-SCI), a tool developed to measure physical activity among people with SCI. ${ }^{17}$ The PARA-SCI has demonstrated reliability and validity as a measure of physical activity through comparisons with indirect calorimetry and doubly labelled water. ${ }^{17-19}$ The PARA-SCI interview protocol was developed using a team of researchers, health-care professionals and people with SCI. The interview is broken down into eight periods over the day (for example, lunch, afternoon, evening routine and so on); question flowcharts were used during the interviews to assist with participant recall. ${ }^{16,18}$ During the structured telephone interview, conducted by trained research assistants in accordance with the PARA-SCI administration guidelines, participants recalled all physical activities performed over the previous 3 days that required exertion, including both LTPA and daily activities. ${ }^{18,20}$ For each activity, participants reported the duration, in minutes, and rated the intensity using validated intensity classification definitions. ${ }^{17}$ For this study, only the daily activities data were analysed. Average minutes per day spent participating in each type of daily activity was calculated by summing the minutes of mild-, moderate- and heavyintensity daily activities reported across the 3 recalled days and dividing by three. The same process was used to calculate the minutes per day of total daily activities.

\section{Demographic and injury-related characteristics}

Age, sex, education, ethnicity and marital status were reported. Participants identified their main mode of mobility used outside of the home, which was subsequently classified as a manual wheelchair, power wheelchair or gait aid. Injury severity was classified by creating a composite variable; the variable was created to align with the data available in the International Spinal Cord Injury Core Data Set. ${ }^{21}$ Accordingly, four groups were created by combining participants' self-reported AIS score and the level of injury. ${ }^{21}$ Participants with an AIS score of $\mathrm{A}-\mathrm{C}$ and injuries between $\mathrm{C} 1$ and $\mathrm{C} 4$ formed the first group. The second group comprised individuals with an AIS score of A-C and injuries between $\mathrm{C} 5$ and $\mathrm{C} 7$. Participants with an AIS score of A-C and paraplegia (that is, T1-S5) formed the third group. The final group of participants was composed of individuals with an AIS score of D, including both paraplegia and tetraplegia.

\section{Data analysis}

Responses were screened for outliers and missing data and tested for normality. Outliers, defined as $Z$-scores greater than an absolute value of 3.29, were replaced with the next highest value in the data set below 3.29 or above $-3.29 .^{22}$ There were 11,14 and 14 outliers for average minutes per day of mild-, moderate- and heavy-intensity daily activities, respectively. As fewer than $5 \%$ of values were missing for minutes per day of total daily activities $(n=6$, $0.86 \%$ ), these observations were deleted. ${ }^{22}$ The total daily activities data deviated from normality and could not be remedied through transformation. Given the robustness of analysis of variance (ANOVA), as well as the limitations of nonparametric analyses, the analyses proceeded using raw data. $^{22}$

To address the first study objective, 37 different types of daily activities were identified in the data set. To ensure adequate sample size, a daily activity was included in the analysis if at least $5 \%$ of the sample $(n=35)$ reported it; 26 daily activities met this criterion and were analysed. For each type of daily activity, a multivariate ANOVA (MANOVA) was conducted to compare minutes per day spent performing that activity at a mild versus moderate versus heavy intensity. Significant MANOVAs were decomposed using three paired-sample $t$-tests. Given the multiple comparisons, a Bonferroni-adjusted $P$-value of 0.017 was used. ${ }^{22}$ Effect sizes were calculated using Cohen's $d$, which were interpreted as small $(0.20)$, medium $(0.50)$ and large $(0.80) \cdot{ }^{23}$ For ease of presentation, daily activities were grouped according to patterns that emerged in the comparisons across intensities. For the second objective, MANOVAs were used to test for differences in activity amounts and intensities as a function of demographic and injury-related characteristics. Significant MANOVAs were decomposed using three one-way ANOVAs with a Bonferroni-adjusted $P$-value of .017 to account for multiple comparisons. Significant ANOVAs were followed with Bonferroni post hoc tests (equal variances assumed) or Tamhane's T2 post hoc tests (equal variances not assumed). ${ }^{22}$ All statistical tests were conducted using SPSS v. 20 (IBM, Markham, ON, Canada).

\section{RESULTS}

\section{Type, intensity and duration of daily activities}

Participants reported an average of $127.92 \pm 142.79 \mathrm{~min}$ per day of total daily activities, with significantly more minutes per day spent on mild-intensity $(78.93 \pm 104.62 \mathrm{~min}$ per day) than moderate-intensity $(40.23 \pm 68.71 \mathrm{~min}$ per day, $t=9.06, P<0.0001)$ or heavy-intensity activities $(8.75 \pm 24.53 \mathrm{~min}$ per day, $t=17.33, P<0.0001)$. More minutes per day were also spent in moderate- versus heavy-intensity daily activities $(t=12.27, P<0.0001)$.

There were four unique patterns of participation across the various daily activities (Tables 1,2,3 and 4). For 11 activities (for example, dressing), significant differences were observed across all three intensities (pattern 1, Table 1) such that significantly more time was spent engaging in these activities at a mild intensity than either moderate or heavy intensities, and significantly more time was spent engaging in these activities at a moderate intensity than a heavy intensity. For eight activities (for example, cleaning), there were no 
Table 1 Significant differences across all three intensities in the mean minutes per day (s.d.) spent in daily physical activities reported in the SHAPE-SCI data set

\begin{tabular}{|c|c|c|c|c|c|c|c|}
\hline $\begin{array}{l}\text { Type of physical } \\
\text { activity }\end{array}$ & $\begin{array}{c}\text { Number (\%) } \\
\text { reporting }\end{array}$ & $\begin{array}{c}\text { Total daily } \\
\text { physical activity }\end{array}$ & Mild intensity & $\begin{array}{l}\text { Moderate } \\
\text { intensity }\end{array}$ & $\begin{array}{l}\text { Heavy } \\
\text { intensity }\end{array}$ & MANOVA & Effect size (Cohen's d) \\
\hline Transfers & $463(66.62)$ & $7.49(10.40)$ & $3.83_{a}(6.37)$ & $2.74_{b}(6.21)$ & $0.92_{c}(3.86)$ & $\begin{array}{l}\mathrm{F}(3,460)=83.69, P<0.0001 \\
\eta^{2}=0.35\end{array}$ & $\begin{array}{l}d_{\text {mild, moderate }}=0.17 \\
d_{\text {mild, heavy }}=0.55 \\
d_{\text {moderate, heavy }}=0.35\end{array}$ \\
\hline Any dressing & $284(40.86)$ & $12.41(13.34)$ & $7.48_{a}(9.73)$ & $3.82_{b}(8.35)$ & $1.10_{c}(5.33)$ & $\begin{array}{l}\mathrm{F}(3,281)=87.83, P<0.0001 \\
\eta^{2}=0.48\end{array}$ & $\begin{array}{l}d_{\text {mild, }} \text { moderate } \\
d_{\text {mild, heavy }}=0.40 \\
d_{\text {moderate, heavy }}=0.39\end{array}$ \\
\hline Wheeling & $282(40.58)$ & 23.32 (48.78) & $14.72_{\mathrm{a}}(38.20)$ & $7.20_{b}(20.15)$ & $1.41_{c}(5.02)$ & $\begin{array}{l}\mathrm{F}(3,279)=27.12, P<0.0001 \\
\eta^{2}=0.23\end{array}$ & $\begin{array}{l}d_{\text {mild, moderate }}=0.25 \\
d_{\text {mild, heavy }}=0.49 \\
d_{\text {moderate, heavy }}=0.39\end{array}$ \\
\hline Toileting & $238(34.24)$ & 26.25 (28.49) & $16.70_{a}(26.27)$ & $6.92_{b}(13.96)$ & $2.63_{c}(12.70)$ & $\begin{array}{l}\mathrm{F}(3,235)=73.00, P<0.0001 \\
\eta^{2}=0.48\end{array}$ & $\begin{array}{l}d_{\text {mild, }} \text { moderate }=0.46 \\
d_{\text {mild, heavy }}=0.68 \\
d_{\text {moderate, heavy }}=0.32\end{array}$ \\
\hline Shower & $181(26.04)$ & 12.69 (12.09) & $8.20_{a}(11.24)$ & $3.21_{b}(7.52)$ & $0.78_{c}(5.35)$ & $\begin{array}{l}F(3,178)=68.60, P<0.0001 \\
\eta^{2}=0.48\end{array}$ & $\begin{array}{l}d_{\text {mild, moderate }}=0.52 \\
d_{\text {mild, heavy }}=0.84 \\
d_{\text {moderate, heavy }}=0.37\end{array}$ \\
\hline Grooming & $151(21.73)$ & $11.13(10.10)$ & $7.90_{a}(8.75)$ & $2.98_{b}(7.02)$ & $0.26_{c}(1.90)$ & $\begin{array}{l}\mathrm{F}(3,148)=61.89, P<0.0001 \\
\eta^{2}=0.56\end{array}$ & $\begin{array}{l}d_{\text {mild, moderate }}=0.62 \\
d_{\text {mild, heavy }}=1.21 \\
d_{\text {moderate, heavy }}=0.53\end{array}$ \\
\hline Desk work & $129(18.56)$ & $\begin{array}{c}118.79 \\
(118.67)\end{array}$ & $\begin{array}{c}83.76 a \\
(105.65)\end{array}$ & $33.28_{b}(81.10)$ & $1.74_{c}(11.70)$ & $\begin{array}{l}\mathrm{F}(3,126)=44.30, P<0.0001 \\
\eta^{2}=0.51\end{array}$ & $\begin{array}{l}d_{\text {mild, moderate }}=0.54 \\
d_{\text {mild, heavy }}=1.09 \\
d_{\text {moderate, heavy }}=0.54\end{array}$ \\
\hline Eating meals & $87(12.52)$ & 29.20 (29.39) & $21.71_{\mathrm{a}}(24.88)$ & $7.48_{b}(22.09)$ & $0.02_{c}(0.18)$ & $\begin{array}{l}\mathrm{F}(3,84)=30.86, P<0.0001 \\
\eta^{2}=0.52\end{array}$ & $\begin{array}{l}d_{\text {mild, moderate }}=0.60 \\
d_{\text {mild, heavy }}=1.23 \\
d_{\text {moderate, heavy }}=0.48\end{array}$ \\
\hline $\begin{array}{l}\text { Dressing upper } \\
\text { body }\end{array}$ & $83(11.94)$ & $5.28(4.72)$ & $3.61_{a}(4.35)$ & $1.52_{b}(3.01)$ & $0.14_{c}(1.12)$ & $\begin{array}{l}F(3,83)=33.75, P<0.0001 \\
\eta^{2}=0.56\end{array}$ & $\begin{array}{l}d_{\text {mild, moderate }}=0.56 \\
d_{\text {mild, heavy }}=1.09 \\
d_{\text {moderate, heavy }}=0.61\end{array}$ \\
\hline Driving & $73(10.50)$ & $36.16(37.10)$ & $27.05_{a}(32.81)$ & $7.51_{b}(15.81)$ & $1.60_{c}(9.31)$ & $\begin{array}{l}\mathrm{F}(3,70)=22.53, P<0.0001 \\
\eta^{2}=0.49\end{array}$ & $\begin{array}{l}d_{\text {mild, moderate }}=0.76 \\
d_{\text {mild, heavy }}=1.06 \\
d_{\text {moderate, heavy }}=0.46\end{array}$ \\
\hline Personal hygiene & $47(6.76)$ & $9.98(6.79)$ & $8.89_{a}(7.22)$ & $1.09_{b}(2.79)$ & $0.00_{c}(0.00)$ & $\begin{array}{l}\mathrm{F}(2,45)=52.07, P<0.0001 \\
\eta^{2}=0.70\end{array}$ & $\begin{array}{l}d_{\text {mild, moderate }}=1.43 \\
d_{\text {mild, heavy }}=1.74 \\
d_{\text {moderate, heavy }}=0.55\end{array}$ \\
\hline
\end{tabular}

Abbreviations: MANOVA, multivariate analysis of variance; SHAPE-SCI, Study of Health and Activity in People with Spinal Cord Injury.

Within each row, different subscripts denote significantly different values $(P<0.017)$.

significant differences in minutes per day of mild- and moderateintensity activity; however, participants accrued significantly fewer minutes per day of heavy-intensity activity than both mild- and moderate-intensity activity (pattern 2, Table 2). Two daily activities, positioning and animal care, fit a third pattern whereby participants reported significantly more minutes per day of mild-intensity than heavy-intensity activity, but there were no differences in minutes per day of mild versus moderate intensity, or moderate versus heavy intensity (pattern 3, Table 3). For five daily activities (for example, lifting), there were no significant differences in the minutes per day performed at each intensity (pattern 4, Table 4).

Group differences on mild, moderate and heavy intensity minutes per day of daily activities

The MANOVAs revealed significant between-group differences in minutes per day of mild, moderate and heavy-intensity daily activities as a function of education, injury severity and mode of mobility (Table 5). There were significant between-group differences for education groups with regard to minutes per day of mild-intensity daily activities, $\mathrm{F}(4,678)=4.53, P<0.01, \eta^{2}=0.03$. Participants with a high school education or lower (that is, 'other') accrued significantly fewer minutes per day of mild-intensity activities when compared with participants with a college education. There were also between-group differences for injury severity categories with regard to minutes per day of heavy-intensity activities, $\mathrm{F}(3,676)=5.41, \quad P<0.01$, $\eta^{2}=0.02$. Participants with an injury classified as AIS A-C, C1-C4 or AIS A-C, T1-S5 reported significantly fewer minutes per day of heavy-intensity activities than those classified as AIS D. There were also between-group differences for mode of mobility with regard to minutes per day of mild, $\mathrm{F}(2,686)=5.96, P<0.01, \eta^{2}=0.02$, and heavy-intensity activities, $\mathrm{F}(2,686)=8.43, \quad P<0.0001, \quad \eta^{2}=0.02$. Manual and power chair users reported significantly more minutes per day of mild-intensity daily activities, but significantly less time on heavy-intensity activities than gait aid users. Contrary to hypotheses, there were no gender differences with respect to the time spent on daily activities.

\section{DISCUSSION}

The objectives of this paper were to describe the type, intensity and duration of daily physical activities reported by people with SCI, and to describe and compare minutes per day spent on daily activities across subgroups. Overall, participants engaged in $\sim 128$ min per day 
Table 2 No significant differences between mild and moderate intensities, significant differences from heavy intensity in mean minutes per day (s.d.) spent in daily activities reported in the SHAPE-SCI data set

\begin{tabular}{|c|c|c|c|c|c|c|c|}
\hline $\begin{array}{l}\text { Type of physical } \\
\text { activity }\end{array}$ & $\begin{array}{l}\text { Number (\%) } \\
\text { reporting }\end{array}$ & $\begin{array}{l}\text { Total daily } \\
\text { physical activity }\end{array}$ & Mild intensity & $\begin{array}{l}\text { Moderate } \\
\text { intensity }\end{array}$ & $\begin{array}{l}\text { Heavy } \\
\text { intensity }\end{array}$ & MANOVA & Effect size (Cohen's d) \\
\hline Cleaning & 208 (29.93) & $31.92(36.14)$ & $\begin{array}{l}16.65 a \\
(24.84)\end{array}$ & $12.39_{a}(22.93)$ & $2.89 \mathrm{~b}(10.85)$ & $\begin{array}{l}\mathrm{F}(3,205)=54.39, P<0.0001 \\
\eta^{2}=0.44\end{array}$ & $\begin{array}{l}d_{\text {mild, }} \text { moderate }=0.18 \\
d_{\text {mild, heavy }}=0.72 \\
d_{\text {moderate, heavy }}=0.53\end{array}$ \\
\hline Dressing lower body & $131(18.85)$ & $8.45(7.66)$ & $4.21_{\mathrm{a}}(5.48)$ & $3.50_{a}(5.50)$ & $0.74_{b}(3.35)$ & $\begin{array}{l}\mathrm{F}(3,128)=53.29, P<0.0001 \\
\eta^{2}=0.55\end{array}$ & $\begin{array}{l}d_{\text {mild, moderate }}=0.13 \\
d_{\text {mild, heavy }}=0.76 \\
d_{\text {moderate, heavy }}=0.61\end{array}$ \\
\hline Walking & 95 (13.97) & $17.77(24.20)$ & $7.48_{a}(15.81)$ & $7.47_{a}(17.66)$ & $2.83_{b}(6.61)$ & $\begin{array}{l}F(3,92)=18.89, P=<0.0001 \\
\eta^{2}=0.38\end{array}$ & $\begin{array}{l}d_{\text {mild, moderate }}=0.00 \\
d_{\text {mild, heavy }}=0.38 \\
d_{\text {moderate, heavy }}=0.35\end{array}$ \\
\hline Bathing & $85(12.23)$ & $14.74(13.56)$ & $8.34_{a}(11.88)$ & $5.74_{a}(10.95)$ & $0.66_{b}(4.46)$ & $\begin{array}{l}\mathrm{F}(3,82)=32.96, P<0.0001 \\
\eta^{2}=0.55\end{array}$ & $\begin{array}{l}d_{\text {mild, }} \text { moderate }=0.23 \\
d_{\text {mild, heavy }}=0.86 \\
d_{\text {moderate, heavy }}=0.61\end{array}$ \\
\hline Food preparation & 72 (10.36) & $16.97(20.14)$ & $\begin{array}{l}10.94 a \\
(18.83)\end{array}$ & $4.88_{a}(10.90)$ & $1.14_{b}(5.21)$ & $\begin{array}{l}F(3,69)=17.42, P<0.0001 \\
\eta^{2}=0.43\end{array}$ & $\begin{array}{l}d_{\text {mild, moderate }}=0.39 \\
d_{\text {mild, heavy }}=0.71 \\
d_{\text {moderate, heavy }}=0.44\end{array}$ \\
\hline Laundry & $64(9.21)$ & 15.99 (18.19) & $\begin{array}{l}10.18 \mathrm{a} \\
(16.91)\end{array}$ & $4.85_{a}(10.37)$ & $0.96_{b}(3.21)$ & $\begin{array}{l}\mathrm{F}(3,61)=18.19, P<0.0001 \\
\eta^{2}=0.47\end{array}$ & $\begin{array}{l}d_{\text {mild, moderate }}=0.38 \\
d_{\text {mild, heavy }}=0.76 \\
d_{\text {moderate, heavy }}=0.51\end{array}$ \\
\hline Opening doors & $60(8.63)$ & $1.33(1.64)$ & $0.64_{a}(1.40)$ & $0.59_{a}(1.20)$ & $0.10_{b}(0.35)$ & $\begin{array}{l}\mathrm{F}(3,57)=13.75, P<0.0001 \\
\eta^{2}=0.42\end{array}$ & $\begin{array}{l}d_{\text {mild, moderate }}=0.04 \\
d_{\text {mild, heavy }}=0.53 \\
d_{\text {moderate, heavy }}=0.55\end{array}$ \\
\hline Doing dishes & $42(6.04)$ & 10.98 (14.28) & $5.42_{a}(10.28)$ & $5.15_{a}(10.87)$ & $0.41_{b}(2.09)$ & $\begin{array}{l}\mathrm{F}(3,39)=8.28, P<0.0001 \\
\eta^{2}=0.39\end{array}$ & $\begin{array}{l}d_{\text {mild, moderate }}=0.03 \\
d_{\text {mild, heavy }}=0.68 \\
d_{\text {moderate, heavy }}=0.61\end{array}$ \\
\hline
\end{tabular}

Abbreviations: MANOVA, multivariate analysis of variance; SHAPE-SCI, Study of Health and Activity in People with Spinal Cord Injury.

Within each row, different subscripts denote significantly different values $(P<0.017)$.

Table 3 No significant differences between mild and moderate or moderate and heavy intensities, significant differences between mild and heavy intensities in mean minutes per day (s.d.) spent in daily activities reported in the SHAPE-SCI data set

\begin{tabular}{|c|c|c|c|c|c|c|c|}
\hline $\begin{array}{l}\text { Type of } \\
\text { physical activity }\end{array}$ & $\begin{array}{l}\text { Number (\%) } \\
\text { reporting }\end{array}$ & $\begin{array}{c}\text { Total daily } \\
\text { physical activity }\end{array}$ & Mild intensity & Moderate intensity & Heavy intensity & MANOVA & Effect size (Cohen's d) \\
\hline Positioning & $149(21.44)$ & $15.94(39.54)$ & $8.76_{a}(24.02)$ & $5.69_{\mathrm{ab}}(22.25)$ & $1.49_{b}(6.65)$ & $\begin{array}{l}\mathrm{F}(3,146)=10.10, P<0.0001 \\
\eta^{2}=0.17\end{array}$ & $\begin{array}{l}d_{\text {mild, moderate }}=0.13 \\
d_{\text {mild, heavy }}=0.41 \\
d_{\text {moderate, heavy }}=0.26\end{array}$ \\
\hline Animal care & $38(5.47)$ & $12.52(16.64)$ & $9.95_{a}(16.33)$ & $2.35_{a b}(7.22)$ & $0.22_{b}(1.35)$ & $\begin{array}{l}\mathrm{F}(3,35)=7.20, P<0.001 \\
\eta^{2}=0.38\end{array}$ & $\begin{array}{l}d_{\text {mild, moderate }}=0.60 \\
d_{\text {mild, heavy }}=0.84 \\
d_{\text {moderate, heavy }}=0.41\end{array}$ \\
\hline
\end{tabular}

Abbreviations: MANOVA, multivariate analysis of variance; SHAPE-SCI, Study of Health and Activity in People with Spinal Cord Injury.

Within each row, different subscripts denote significantly different values $(P<0.017)$.

of daily activities (not including LTPA), reporting more mild- versus moderate- and more moderate- versus heavy-intensity activities. When looking at the intensity at which daily activities were performed, four patterns of participation emerged, indicating that some types of activities were performed mostly at a mild intensity (for example, dressing and wheeling), whereas other activities were performed equally at mild and moderate intensities (for example, cleaning and walking), or equally at moderate and heavy intensities (for example, positioning). Furthermore, time spent engaging in mild, moderate and heavy daily activities varied as a function of education, injury severity and mode of mobility. Below we expand upon these observations.

Accumulating evidence suggests that, for adults with SCI to achieve fitness benefits, physical activity must be performed at a moderate or heavy intensity. ${ }^{15}$ If daily activities are to be encouraged as a means to increase overall physical activity levels in people with SCI, then it is important to identify and promote daily activities that are typically performed at intensities conducive to fitness gains. Our study revealed that, overall, participants spent more time in mild- than moderate- or heavy-intensity daily activities. However, consistent with previous research, ${ }^{13}$ we found variability in the intensity at which different types of activities were typically performed. Furthermore, although most daily activities resulted in relatively small accruals of total activity (that is, $<20 \mathrm{~min}$ per day), a few activities_cleaning, yard work and making repairs-accrued larger numbers of minutes per day of total activity and moderate-intensity activity. As these are activities that people with SCI seem to do at greater intensities and for longer durations, ${ }^{12}$ they may be ideal daily activities to promote as a means to accrue greater total daily activity. 
Table 4 No significant differences in minutes spent across intensities in mean minutes per day (s.d.) spent in daily activities reported in the SHAPE-SCI data set

\begin{tabular}{|c|c|c|c|c|c|c|c|}
\hline $\begin{array}{l}\text { Type of physical } \\
\text { activity }\end{array}$ & $\begin{array}{l}\text { Number (\%) } \\
\text { reporting }\end{array}$ & $\begin{array}{c}\text { Total daily } \\
\text { physical activity }\end{array}$ & Mild intensity & Moderate intensity & Heavy intensity & MANOVA & Effect size (Cohen's d) \\
\hline Lifting & $89(12.81)$ & $13.86(38.03)$ & $4.51(9.42)$ & 7.67 (36.53) & $1.68(8.70)$ & $\begin{array}{l}\mathrm{F}(3,86)=9.69, P<0.0001 \\
\eta^{2}=0.25\end{array}$ & $\begin{array}{l}d_{\text {mild, }} \text { moderate }=0.12 \\
d_{\text {mild, heavy }}=0.31 \\
d_{\text {moderate, heavy }}=0.23\end{array}$ \\
\hline Carrying & $58(8.35)$ & $13.19(20.94)$ & $4.18(8.93)$ & 7.05 (18.65) & $1.96(6.86)$ & $\begin{array}{l}\mathrm{F}(3,55)=9.35, P<0.0001 \\
\eta^{2}=0.34\end{array}$ & $\begin{array}{l}d_{\text {mild, moderate }}=0.20 \\
d_{\text {mild, heavy }}=0.28 \\
d_{\text {moderate, heavy }}=0.36\end{array}$ \\
\hline Yard work & $45(6.47)$ & $50.96(62.10)$ & $23.11(38.58)$ & $19.70(44.92)$ & $8.16(25.62)$ & $\begin{array}{l}F(3,42)=10.18 \\
P<0.0001, \eta^{2}=0.42\end{array}$ & $\begin{array}{l}d_{\text {mild, moderate }}=0.08 \\
d_{\text {mild, heavy }}=0.46 \\
d_{\text {moderate, heavy }}=0.32\end{array}$ \\
\hline Grocery shopping & $43(6.19)$ & $10.10(9.27)$ & $4.88(6.09)$ & $3.71(7.45)$ & $1.51(6.45)$ & $\begin{array}{l}\mathrm{F}(3,40)=19.95 \\
P<0.0001, \eta^{2}=0.60\end{array}$ & $\begin{array}{l}d_{\text {mild, moderate }}=0.17 \\
d_{\text {mild, heavy }}=0.54 \\
d_{\text {moderate, heavy }}=0.32\end{array}$ \\
\hline Repairs & $43(6.19)$ & $44.67(55.29)$ & $20.5839 .40)$ & $14.24(27.71)$ & $9.84(35.54)$ & $\begin{array}{l}\mathrm{F}(3,40)=10.41 \\
P<0.0001, \eta^{2}=0.44\end{array}$ & $\begin{array}{l}d_{\text {mild, }} \text { moderate }=0.18 \\
d_{\text {mild, heavy }}=0.29 \\
d_{\text {moderate, heavy }}=0.14\end{array}$ \\
\hline
\end{tabular}

Abbreviations: MANOVA, multivariate analysis of variance; SHAPE-SCI, Study of Health and Activity in People with Spinal Cord Injury.

Interestingly, although previous research involving people with SCI suggests that mobility-related daily activities (for example, transferring and wheeling) result in considerable energy expenditure and may have health benefits, ${ }^{12,13}$ study participants spent relatively little time on these activities. Also, these daily activities were performed for longer durations at mild, than moderate or heavy intensities. Given the potential benefits of mobility-related daily activities, there may be utility in encouraging people with SCI to increase the time and effort they spend on these activities.

With respect to the second objective, as hypothesised, time spent on mild-, moderate- and heavy-intensity daily activities differed across groups based on modes of mobility and levels of injury severity. Specifically, gait aid users accrued significantly fewer minutes per day of mild-intensity daily activities than both power and manual wheelchair users, but more minutes per day of heavy-intensity activities than either group. One possible explanation for this observation is that walking with gait aids is more metabolically demanding than using a manual or power chair, and, as a result, gait aid users would report more activity at heavier intensities..$^{24,25}$ It is also possible that participants who use a gait aid outside of the home may have the functional capacity and energy to engage in more intense forms of daily activities, such as lifting and carrying, when compared with manual and power chair users. Furthermore, with respect to manual chair users, studies have found that greater wheelchair skill is associated with greater overall social participation. ${ }^{26,27}$ Thus, it is possible that poorer wheelchair skills may result in more time needed to complete various daily activities. Future studies of daily activities should not only consider mode of mobility as a moderator but also participants' physical function and skill using their mobility device.

Participants classified as AIS D reported two to three times more minutes per day of heavy-intensity daily activities when compared with participants with AIS A-C high tetraplegia and AIS A-C paraplegia. Similar findings exist for LTPA, such that people with any tetraplegia accrue significantly fewer minutes per day of LTPA than participants classified as AIS D. ${ }^{6}$ Our results deviated slightly from these studies, such that the minutes per day difference between participants classified as AIS D and participants with low tetraplegia (AIS A-C, C5-C7) was not statistically significant. In comparison with participants with high tetraplegia (AIS A-C, C1-C4), participants with low tetraplegia may have more function and may be more likely to use a manual chair, which could afford more opportunities for more intense forms of daily activities such as wheeling. However, in comparison with participants with paraplegia (AIS A-C), participants with low tetraplegia may have more functional impairments that make such activities (for example, transfers and cleaning) feel more intense and take longer to complete.

Contrary to our hypothesis, there were significant differences in minutes per day spent engaging in various intensities of daily activities with respect to education and gender. Participants from the high school and 'other' categories reported engaging in fewer minutes per day of mild-intensity activity than those with a college diploma. Education may be a proxy for socioeconomic status, which has been associated with health disparities including access to and use of physical activity facilities, income from employment and access to built environments that facilitate activity. ${ }^{28,29}$ Thus, it is possible that higher education is associated with more mild- and moderate-intensity daily activities because these participants have more opportunities to engage in daily activities through employment and the income it provides, and environments conductive to such activity. With respect to gender, it is possible that differences between groups disappear given that daily activities incorporate all activities outside of LTPA and sedentary time. Thus, although past research suggests that women may spend more time on domestic daily activities such as cleaning and food preparation, ${ }^{11}$ it is possible that men may spend more time on other types of daily activities, such as building.

\section{Limitations}

Our study is among the first to explore and describe alternative sources of physical activity among people with SCI; however, this study is not without its limitations. Although the PARA-SCI is a valid and reliable self-report measure of physical activity, ${ }^{17-19}$ there may be a risk of recall bias. Furthermore, it should be noted that, in many instances, small sample sizes and large standard deviations for specific daily activities likely contributed to non-significant $P$-values in Pattern 4, where there were no significant differences between intensities. Further investigation of certain types of daily activities 
Table 5 Total minutes per day of total daily activities as a function of demographic and injury-related characteristics

\begin{tabular}{|c|c|c|c|c|c|c|}
\hline Characteristic & $\begin{array}{c}\text { Number } \\
\text { (\%) }\end{array}$ & $\begin{array}{c}\text { Total minutes } \\
\text { (s.d.) }\end{array}$ & $\begin{array}{l}\text { Mild intensity } \\
\text { (s.d.) }\end{array}$ & $\begin{array}{c}\text { Moderate intensity } \\
\text { (s.d.) }\end{array}$ & $\begin{array}{l}\text { Heavy intensity } \\
\text { (s.d.) }\end{array}$ & MANOVA \\
\hline \multicolumn{7}{|l|}{ Sex } \\
\hline Female & $161(23.37)$ & $138.19(132.16)$ & $81.67(89.83)$ & $44.48(69.00)$ & $12.04(29.98)$ & $\mathrm{F}(3,685)=1.34, \mathrm{NS}, \eta^{2}=0.006$ \\
\hline Male & $528(76.63)$ & $124.79(145.86)$ & $78.10(108.80)$ & $38.94(68.64)$ & $7.75(22.54)$ & \\
\hline \multicolumn{7}{|l|}{ Education $^{\mathrm{a}}$} \\
\hline High school & 239 (34.99) & $117.50(127.65)$ & $63.82_{a}(77.59)$ & $42.96(69.11)$ & $10.71(29.51)$ & $\mathrm{F}(12,2034)=2.52, P<0.01, \eta^{2}=0.015$ \\
\hline College & $167(24.45)$ & $139.27(158.48)$ & $99.28_{b}(132.86)$ & $32.01(53.10)$ & $7.99(21.91)$ & \\
\hline University & $120(17.57)$ & $143.84(160.64)$ & $95.93_{\mathrm{ab}}(120.03)$ & $42.70(80.94)$ & $5.21(16.98)$ & \\
\hline Postgraduate & $45(6.59)$ & $125.45(133.66)$ & $79.46_{a b}(90.76)$ & $39.62(80.34)$ & $6.37(16.89)$ & \\
\hline Other & $112(16.40)$ & $116.42(133.55)$ & $61.07 \mathrm{a}(85.93)$ & $45.48(70.79)$ & $9.87(24.50)$ & \\
\hline \multicolumn{7}{|l|}{ Ethnicity } \\
\hline Caucasian & $611(89.33)$ & $127.81(140.76)$ & $79.50(104.65)$ & $40.04(67.74)$ & $8.26(22.60)$ & $F(12,2037)=0.84, N S, \eta^{2}=0.005$ \\
\hline Native Canadian & 17 (2.49) & $125.31(205.08)$ & $90.17(168.40)$ & $29.91(50.86)$ & $5.23(8.38)$ & \\
\hline Black & $20(2.92)$ & 102.25 (117.87) & $52.95(66.47)$ & $42.72(67.02)$ & $6.58(12.95)$ & \\
\hline Asian & $16(2.34)$ & 139.33 (177.75) & $63.62(83.81)$ & $52.46(119.30)$ & $23.25(52.86)$ & \\
\hline Other & $20(2.92)$ & $141.99(156.65)$ & $80.74(76.79)$ & $47.27(71.71)$ & $13.98(46.53)$ & \\
\hline \multicolumn{7}{|l|}{ Marital status } \\
\hline Single & $242(35.48)$ & $116.64(133.72)$ & $78.70(107.12)$ & $30.90(53.55)$ & $7.04(23.14)$ & $\mathrm{F}(12,2031)=1.01, \mathrm{NS}, \eta^{2}=0.006$ \\
\hline Married & 304 (44.57) & $131.86(146.82)$ & 76.04 (97.37) & $45.67(75.88)$ & $10.15(27.59)$ & \\
\hline Common law & $35(5.13)$ & $140.64(147.32)$ & 90.95 (101.93) & $42.10(81.45)$ & 7.59 (15.25) & \\
\hline Divorced & $86(12.61)$ & 139.56 (158.87) & $81.51(124.82)$ & $50.20(78.56)$ & 7.85 (18.21) & \\
\hline Widowed & $15(2.20)$ & 126.09 (116.83) & $92.08(93.45)$ & $24.88(27.16)$ & 9.12 (19.75) & \\
\hline \multicolumn{7}{|l|}{ Injury severity ${ }^{\mathrm{a}}$} \\
\hline C1-C4, Asia A-C & $75(11.03)$ & $136.64(155.10)$ & $95.06(123.07)$ & $36.65(83.32)$ & $4.93_{a}(16.14)$ & $\mathrm{F}(9,2028)=2.85, P<0.01, \eta^{2}=0.012$ \\
\hline C5-C7, Asia A-C & $182(26.76)$ & $142.74(158.22)$ & $88.87(111.48)$ & $45.45(69.84)$ & $8.41_{\mathrm{ab}}(23.58)$ & \\
\hline T1-S5, Asia A-C & 252 (37.06) & 114.05 (137.66) & $75.18(104.86)$ & $32.74(61.64)$ & $6.14_{a}(17.83)$ & \\
\hline Asia D, Any level & $171(25.15)$ & $132.38(128.33)$ & $68.94(88.04)$ & $48.35(71.06)$ & $15.08_{b}(34.63)$ & \\
\hline \multicolumn{7}{|l|}{ Mode of mobility ${ }^{a}$} \\
\hline Manual chair & $385(55.88)$ & $122.15(135.21)$ & $76.57_{a}(93.86)$ & $37.91(67.22)$ & $7.68_{a}(20.66)$ & $\mathrm{F}(6,1370)=2.85, P<0.001, \eta^{2}=0.022$ \\
\hline Power chair & $220(31.93)$ & $141.68(162.47)$ & $94.39 \mathrm{a}(127.76)$ & $40.52(71.51)$ & $6.77_{a}(21.48)$ & \\
\hline Gait aid & $84(12.19)$ & $118.31(118.45)$ & $49.27_{b}(73.19)$ & $50.15(67.93)$ & $18.88_{b}(40.91)$ & \\
\hline
\end{tabular}

Abbreviation: MANOVA, multivariate analysis of variance.

Some participants did not respond to a specific demographic question; thus, the sum of participants may not add up to 695 for some demographic variables.

a Different lower case subscripts denote significantly different values between demographic groups for a specific intensity $(P<0.017)$

(for example, lifting and yard work) is warranted. Given our crosssectional analysis was limited to descriptive statistics and group comparisons, we cannot ascertain the impact that various types, durations and intensities of daily activities will have on fitness and health. Future research that examines the association between these daily activities and various health indicators will help inform the viability of daily activities as part of an active lifestyle that enhances the health and well-being of people with SCI. Finally, given that employment status was not measured, it is possible that the findings related to education may actually be more indicative of the relationship between employment and daily activities. Further examination of this relationship is warranted.

\section{Implications}

Although much of the current literature is focused on LTPA, emerging evidence in both the general population and among people with SCI suggests that LTPA on its own may not be enough to reduce chronic disease among people with SCI. ${ }^{30,31}$ Rather prolonged bouts of inactivity may counterbalance the health benefits associated with
LTPA. Given that people with SCI spend the majority of their days inactive or in very low activity (for example, sitting and reading), ${ }^{32}$ this study makes an important contribution to our understanding of what activities people with SCI do between bouts of LTPA and inactivity, as well as assists clinicians to identify patients who may be less likely to be involved in other daily activities that break up inactive time.

As Verschuren et al. ${ }^{33}$ highlight, little is known about sedentary behaviour and energy expenditure in general among people with SCI. Thus, although our study provides a first step in understanding these relationships, future research should examine the association between chronic disease and patterns of daily activities, sedentary behaviour and LTPA. Once these relationships are established, developing interventions aimed at subpopulations most at risk will be necessary.

\section{CONCLUSION}

Given that few people with SCI engage in LTPA, and a small body of literature that suggests an association between daily activities and fitness and health, encouraging daily activities that are carried out at 
greater intensities and for longer durations may be one possible strategy for increasing overall physical activity. Participants engaged in $\sim 128$ min per day of daily activities and most time was spent engaging in mild-intensity activities; moreover, certain demographic/injurycharacteristic groups were more likely to accrue minutes of total daily moderate and heavy-intensity activities than others. Future research is warranted to explore the health-related and psychosocial benefits of engaging in moderate- and heavy-intensity daily activities, as well as targeted interventions to increase daily activity participation among subgroups of people with SCI.

\section{DATA ARCHIVING}

There were no data to deposit.

\section{CONFLICT OF INTEREST}

The authors declare no conflict of interest.

\section{ACKNOWLEDGEMENTS}

MJP was supported by a Social Sciences and Humanities Research Council PDF (756-2013-0531). Funding for the original SHAPE-SCI study was provided by the Canadian Institutes of Health Research (grant no. MOP 57778 awarded to $\mathrm{KMG}$ ).

1 Cragg JJ, Noonan VK, Dvorak M, Krassioukov A, Mancini GBJ, Borisoff JF. Spinal cord injury and type 2 diabetes: results from a population health survey. Neurology 2013; 81: 1864-1868.

2 Myers J, Lee M, Kiratli J. Cardiovascular disease in spinal cord injury: An overview of prevalence, risk, evaluation, and management. Am J Phys Med Rehab 2007; 86: $1-11$.

3 Buchholz AC, Martin Ginis KA, Bray SR, Craven BC, Hicks AL, Hayes KC et al. Greater daily leisure time physical activity is associated with lower chronic disease risk in adults with spinal cord injury. Appl Physiol Nutr Me 2009; 34: 640-647.

4 D'Oliveira GLC, Figueiredo FA, Passos MCF, Chain A, Bezerra FF, Koury JC. Physical exercise is associated with better fat mass distribution and lower insulin resistance in spinal cord injured individuals. J Spinal Cord Med 2014; 37: 79-84.

5 Koury JC, Passos MCF, Figueiredo FA, Chain A, Franco JG. Time of physical exercise practice after injury in cervical spinal cord-injured men is related to the increase in insulin sensitivity. Spinal Cord 2013; 51: 116-119.

6 Martin Ginis KA, Latimer AE, Arbour-Nicitopoulos KP, Buchholz AC, Bray SR, Craven $\mathrm{BC}$ et al. Leisure time physical activity in a population-based sample of people with spinal cord injury part I: demographic and injury-related correlates. Arch Phys Med Rehab 2010; 91: 722-728.

7 Tasiemski T, Bergstrom E, Savic G, Gardner BP. Sports, recreation and employment following spinal cord injury-a pilot study. Spinal Cord 2000; 38: 173-184.

8 US Department of Health and Human Services. Healthy People 2020 Objective Data Search. 2014. Available at http://www.healthypeople.gov/2020/data-search/ Search-the-Data. Last accessed on 1 December 2015.

9 Williams TL, Smith B, Papathomas A. The barriers, benefits and facilitators of leisure time physical activity among people with spinal cord injury: a meta-synthesis of qualitative findings. Health Psychol Rev 2014; 8: 404-425.

10 Martin Ginis KA, Jorgensen S, Stapleton J. Exercise and sport for persons with spinal cord injury. PM R 2012; 4: 894-900.
11 Hetz S, Latimer AE, Martin Ginis KA. Activities of daily living performed by individuals with $\mathrm{SCl}$ : relationships with physical fitness and leisure time physical activity. Spinal Cord 2009; 47: 550-554.

12 Hetz SP, Latimer AE, Buchholz AC, Martin Ginis KA. Increased participation in activities of daily living is associated with lower cholesterol levels in people with spinal cord injury. Arch Phys Med Rehab 2009; 90: 1755-1759.

13 Collins EG, Gater D, Kiratli J, Butler J, Hanson K, Langbein WE. Energy cost of physical activities in persons with spinal cord injury. Med Sci Sport Exer 2010; 42: 691-700.

14 Martin Ginis KA, Mcllraith A, Pomerleau P, Smith K, Stone JA, Wolfe DL et al. The development of evidence-informed physical activity guidelines for adults with spinal cord injury. Spinal Cord 2011; 49: 1088-1096.

15 Hicks AL, Martin Ginis KA, Pelletier CA, Ditor DS, Foulon B, Wolfe DL. The effects of exercise training on physical capacity, strength, body composition and functional performance among adults with spinal cord injury: a systematic review. Spinal Cord 2011; 49: 1103-1127.

16 Martin Ginis KA, Latimer AE, Buchholz AC, Bray SR, Craven BC, Hayes KC et al. Establishing evidence-based physical activity guidelines: methods for the Study of Health and Activity in People with Spinal Cord Injury (SHAPE SCI). Spinal Cord 2008; 46: $216-221$

17 Latimer AE, Martin Ginis KA, Craven BC, Hicks AL. The physical activity recall assessment for people with spinal cord injury: validity. Med Sci Sport Exer 2006; 38: 208-216.

18 Martin Ginis KA, Latimer AE, Hicks AL, Craven BC. Development and evaluation of an activity measure for people with spinal cord injury. Med Sci Sport Exer 2005; 37: 1099-1111.

19 Tanhoffer RA, Tanhoffer AIP, Raymond J, Hills AP, Davis GM. Comparison of methods to assess energy expenditure and physical activity in people with spinal cord injury. J Spinal Cord Med 2012; 35: 35-45.

20 Martin Ginis KA, Latimer AE. Physical Activity Recall Assessment for People with Spinal Cord Injury: Administration and scoring manual. McMaster University, Hamilton, Ontario, Canada. 2008.

21 DeVivo M, Biering-Sørensen F, Charlifue S, Noonan V, Post M, Stripling T et al. International spinal cord injury core data set. Spinal Cord 2006; 44: 535-540.

22 Tabachnick BG, Fidell LS. Using Multivariate Statistics. Pearson/Allyn \& Bacon, Boston, MA, USA. 2007

23 Cohen J. Statistical power analysis for the behavioral sciences. Lawrence Earlbaum Associates, Hillsdale, NJ, USA. 1988.

24 Merati G, Sarchi P, Ferrarin M, Pedotti A, Veicsteinas A. Paraplegic adaptation to assisted-walking: energy expenditure during wheelchair versus orthosis use. Spinal Cord 2000; 38: 37-44.

25 Bateni H, Maki BE. Assistive devices for balance and mobility: benefits, demands, and adverse consequences. Arch Phys Med Rehab 2005; 86: 134-145.

26 Phang SH, Martin Ginis KA, Routhier F, Lemay V. The role of self-efficacy in the wheelchair skills-physical activity relationship among manual wheelchair users with spinal cord injury. Disabil Rehabil 2012; 34: 625-632.

27 Smith EM, Sakakibara BM, Miller WC. A review of factors influencing participation in social and community activities for wheelchair users. Disabil Rehabil Assist Technol 2014; 11: 1-14.

28 Gordon-Larsen P, Nelson MC, Page P, Popkin BM. Inequality in the built environment underlies key health disparities in physical activity and obesity. Pediatrics 2006; 117: 417-424.

29 Ross CE, Wu C. The links between education and health. Am Sociol Rev 1995; 60: 719-745.

30 Totosy de Zepetnek JO, Pelletier CA, Hicks AL, MacDonald MJ. Following the physical activity guidelines for adults with spinal cord injury for 16 weeks does not improve vascular health: a randomized controlled trial. Arch Phys Med Rehabil 2015; 96 : 1566-1575.

31 Van der ploeg HP, Chey T, Korda RJ, Banks E, Bauman A Sitting time and all-cause mortality risk in 222497 Australian adults. Arch Intern Med 2012; 172: 494-500.

32 Perrier MJ, Martin Ginis KA. A description and estimate of very low-intensity activity and inactive awake time in community-dwelling adults with chronic spinal cord injury. Spinal Cord (e-pub ahead of print 19 January 2016; doi:10.1038/sc.2015.2032).

33 Vershuren O, Dekker B, van Koppenhagen C, Post M. Sedentary behavior in people with spinal cord injury. Arch Phys Med Rehab 2016; 97: 173. 